\title{
Bat survey of the lower Juruena River and five new records for the state of Mato Grosso, Brazil
}

\author{
Júlio C. DALPONTE ${ }^{1}$, Renato GREGORIN²*, Vanderléia A. ESTEVES-COSTA³ ${ }^{3}$ Ednaldo C. ROCHA ${ }^{4}$, \\ Rodrigo MARCELINO5 \\ ${ }^{1}$ Instituto para a Conservaçăo dos Carnívoros Neotropicais (PRO-CARNIVOROS). Avenida Horácio Netto, 1.030, Parque Edmundo Zanoni, CEP 12945-010, Atibaia, SP, Brasil. \\ ${ }^{2}$ Universidade Federal de Lavras, Lavras, MG, Brasil. Cx. P. 2037. CEP 37200-000. \\ ${ }^{3}$ Universidade do Estado de Mato Grosso, Av. Tancredo Neves, 1095, Cavalhada II. Cáceres, MT, Brasil. CEP 78200-000. \\ ${ }^{4}$ Universidade Estadual de Goiás, Unidade Universitária de Ipameri. Rodovia GO-330, Km 241. Anel Viário S/N, Setor Universitário. Ipameri, GO, Brasil. CEP 75780-000. \\ 5 Instituto Centro de Vida. Av. Ariosto da Riva Neto, 3473, Centro, Alta Floresta, MT, Brasil. CEP 78580-000. \\ * Corresponding author: rgregorin@dbi.ufla.br
}

\section{ABSTRACT}

The northern Brazilian state of Mato Grosso is considered an important biogeographical region, but has many sampling gaps. Apart from the well-documented non volant mammal community in the region, the bat fauna still poorly recorded. The aim of this study was to record the bat species of Juruena National Park, northern Mato Grosso, Brazil. Nineteen sites were sampled using mist-nets placed at ground level and near potential bat roosts. We collected 115 individuals belonging to 35 species and five families, which increased the number of species known for Mato Grosso's Amazon from 86 to 91 . The five new records were: Peropteryx kappleri, Peropteryx leucoptera, Lonchorhina inusitata, Tonatia saurophila, and Artibeus concolor. Our results pointed out the necessity of more studies in order to better estimate the bat diversity in northern Mato Grosso.

KEYWORDS: Chiroptera, diversity, range extension, southern Amazon

\section{Fauna de morcegos no baixo Rio Juruena, com cinco novos registros para o estado do Mato Grosso, Brasil}

\section{RESUMO}

O norte do estado do Mato Grosso é uma importante região para estudos biogeográficos com muitos hiatos de amostragem sendo a fauna de morcegos pobremente conhecida. O objetivo deste estudo foi fornecer uma lista de morcegos para o Parque Nacional do Juruena, norte do Mato Grosso, Brasil. Foram amostrados 19 sítios e empregadas redes-de-neblina ao nível do solo e próximos a abrigos potenciais de morcegos. Foram coletados 115 indivíduos representando 35 espécies e cinco famílias, aumentando de 86 para 91 o número de espécies conhecidas para a Amazônia no Mato Grosso. Houve cinco novos registros para a porção amazônica do estado: Peropteryx kappleri, Peropteryx leucoptera, Lonchorhina inusitata, Tonatia saurophila e Artibeus concolor. Os resultados indicam a necessidade de mais estudos para uma melhor estimativa da diversidade no norte do Mato Grosso.

PALAVRAS-CHAVE: Chiroptera, diversidade, extensão de distribuição, sul da Amazônia 
The southern and western Brazilian Amazon has long been considered a high priority area for scientific investigations, including basic information about its biodiversity (Bernard $e t$ al. 2011a; Louzada et al. 2015). Southern Brazilian Amazonia encompasses basically the southern portions of the states of Amazonas and Pará, and northern Mato Grosso. Since the 1970 's this area has been subject to a variety of anthropogenic impacts, including logging, mining, and the expansion of commercial and subsistence agriculture (Ferreira et al. 2014). Mato Grosso has lost approximately 13 million hectares of forest (35\% of a total of 37 million hectares deforested in the Brazilian Amazônia) from 2001 to 2009 (MDIC 2012).The rapid regional expansion of human activities contrasts strongly with the paucity of data on biological communities in the area (Bernard et al.2011a). As a result of the few studies conducted there, northern Mato Grosso was considered to have about 11 to 22 species of bats (Miranda-Ribeiro 1914; Bernard et al. 2011a). Recently, and applying a higher sampling effort, Miranda et al. (2015) recorded 33 species along the middle Teles Pires River, which is a more realistic bat richness for an Amazonian site in Mato Grosso State.

Here we present data on a bat survey conducted during a REA (Rapid Ecological Assessment) in Juruena National Park (PNJu), northern Mato Grosso, which includes five new bat records for the state. PNJu has 1.9 million hectares and encompasses the municipalities of Apiacás, Nova Bandeirantes, Colniza and Cotriguaçu (Mato Grosso State), and Apuí and Maués (Amazonas State). The study site $\left(8^{\circ} 55^{\prime} 35.32^{\prime \prime} \mathrm{S}, 58^{\circ} 34^{\prime} 27.75^{\prime \prime} \mathrm{W}\right)$ is located in a transitional zone between the rainforest of the Amazon biome and the savannah of the Cerrado biome.

The region is characterized by a flat topography with small patches of hilly terrain (elevation ranges from $40 \mathrm{~m}$ to $457 \mathrm{~m}$, mean of $185 \mathrm{~m}$ a.s.l.). PNJu lies mostly within the Tapajós River basin, which is formed by two secondary sub-basins: the Juruena and Teles Pires-Arinos, but includes a small part of the Madeira River basin (Santos and Irgang 2008). The Park is covered predominantly with terra firme dense forest and flooded alluvial ombrophylous forests. Small patches of savannah and seasonal semi-deciduous forest also occur. An equatorial climate dominates the region, with temperatures between $24^{\circ} \mathrm{C}$ and $26^{\circ} \mathrm{C}$. Rains are abundant and regular, with annual rainfall ranging from 2,000 to $2,500 \mathrm{~mm}$ during a short dry season (June to September) and a rainy season from October to April. The annual mean humidity varies between $80 \%$ and $90 \%$ (ICMBio 2011).

Bats were sampled from 12 to 28 November 2007 and from 25 February to 15 March 2008, both during the rainy season. Bats were captured with mist nets placed in the understory, between flooded and terra-firme forests. Nets were operated on average for two nights per study site (19 study sites sampled; Table 2), totaling 38 sampled nights. Three seven meter nets were operated from 18:00 h until 00:00 $\mathrm{h}$ and total capture effort was 4,872 net-meter-hours. Some individuals were collected by searching diurnal roosts in tree cavities, foliage, culverts, and abandoned buildings. Bats were collected following the protocols of Sikes et al. (2011). Captures occurred under permission number SISBIO \# 12171. Voucher specimens are presently housed at the Mammal Collection of the Universidade Federal de Lavras (CMUFLA) (Appendix 1).

A total of 115 individuals were collected (Table 1). Of the total collected specimens, 82 were captured in mist nets and 33 were captured in roosts (Tables 1 and 2). Thirty-five species from five families were recorded (Table 1), which is a number slightly higher than Miranda et al. (2015) (33 species and five families) for the middle Teles Pires River (MT). As in many other Amazonian bat inventories (e.g., Simmons and Voss 1998; Lim et al. 2005; Miranda et al. 2015) the most diverse groups recorded were frugivore phyllostomids (14 species), animalivore phyllostomids (nine species), and insectivorous emballonurids (six species). Among the recorded species there are five new records for the state of Mato Grosso and a range extension for the southern Amazon basin, increasing the bat diversity from 86 (Bernard et al. 2011b; Louzada et al. 2015) to 91 species (Table 1) for Amazonian Mato Grosso. The new records are: Peropteryx kappleri, P. leucoptera, Lonchorbina inusitata, Tonatia saurophila, and Artibeus concolor. Though Bernard et al. (2011b) included Carollia castanea in their list of bats for the Brazilian Amazon, Nogueira et al. (2014) excluded that species from the Brazilian list and we therefore do not consider it to be present in Mato Grosso. Regarding the new records for the state of Mato Grosso, the closest record of P. kappleri is Beni (Bolivia) roughly 1,000 km southwest of PNJu; for P. leucoptera, Alter do Chão (Pará, Brazil) is $850 \mathrm{~km}$ northeast; for L. inusitata, Porto Velho (Rondônia, Brazil) is about $560 \mathrm{~km}$ west; for A. concolor, Altamira, Xingu river (Pará, Brazil) is $930 \mathrm{~km}$ northeast; and for T. saurophila, Cachoeira Nazaré (Rondônia, Brazil) is about $370 \mathrm{~km}$ west (Gardner 2008).

There were three specimens of Platyrrhinus angustirostris collected from here that were first mentioned in Nogueira et al. (2014) after identification confirmation by P. Velazco. The specimens had most of the diagnostic characters such as U-shaped uropatagium, metacarpal V longer than IV, and three stylar cusps on PM4. Some traits, however, were distinct from the original description of the species (Velazco et al. 2010), in particular the reduced number of vibrissae (six instead eight) around the noseleaf.

PNJu has a strategic position in the Corredor de Conservaçáo da Amazônia Meridional, ensuring environmental connectivity between protected areas in the far north of Mato 
Table 1. List of bat species recorded to Juruena River, Mato Grosso State, Brazil. Roost = active search on the roosts; net = captured by mist-nets. Nomenclature and classificatory arrangement follows Nogueira et al. (2014).

\begin{tabular}{|c|c|c|c|c|c|}
\hline Taxa & $\mathrm{N}$ & Capture & Taxa & $\mathrm{N}$ & Capture \\
\hline Family Emballonuridae & & & Subfamily Carolliinae & & \\
\hline \multirow{2}{*}{ Peropteryx kappleri Peters, 1867* } & \multirow{2}{*}{3} & \multirow{2}{*}{$\begin{array}{l}\text { roost (hollow } \\
\text { logs) }\end{array}$} & Carollia benkeithi Solari \& Baker, 2006 & 2 & net \\
\hline & & & Carollia brevicauda (Schinz, 1821) & 5 & net \\
\hline Peropteryx leucoptera Peters, $1867^{*}$ & 1 & $\begin{array}{l}\text { roost (hollow } \\
\text { logs) }\end{array}$ & Carollia perspicillata (Linnaeus, 1758) & 15 & net \\
\hline \multirow{2}{*}{ Peropteryx macrotis (Wagner, 1843) } & \multirow{2}{*}{10} & \multirow{2}{*}{$\begin{array}{l}\text { roost (hollow } \\
\text { logs) }\end{array}$} & Subfamily Rhinophyllinae & & \\
\hline & & & Rhinophylla pumilio Peters, 1865 & 2 & net \\
\hline \multirow{2}{*}{ Rhynchonycteris naso (Wied-Neuwied, 1820) } & \multirow[b]{2}{*}{3} & \multirow{2}{*}{$\begin{array}{l}\text { roost (leaning } \\
\text { tree trunk over } \\
\text { water) }\end{array}$} & Subfamily Stenodermatinae & & \\
\hline & & & Artibeus concolor Peters, 1865* & 1 & net \\
\hline \multirow{2}{*}{ Saccopteryx bilineata (Temminck, 1838) } & \multirow{2}{*}{6} & \multirow{2}{*}{$\begin{array}{l}\text { roost (buttresses } \\
\text { in tree trunks) }\end{array}$} & Artibeus lituratus (Olfers, 1818) & 9 & net \\
\hline & & & Artibeus obscurus (Schinz, 1821) & 4 & net \\
\hline \multirow[b]{2}{*}{ Saccopteryx leptura (Schreber, 1774) } & \multirow[b]{2}{*}{1} & \multirow{2}{*}{$\begin{array}{l}\text { roost (over } \\
\text { standing tree } \\
\text { trunk) }\end{array}$} & Artibeus planirostris (Spix, 1823) & 5 & net \\
\hline & & & Chiroderma trinitatum Goodwin, 1958 & 1 & net \\
\hline \multicolumn{3}{|l|}{ Family Phyllostomidae } & Chiroderma villosum Peters, 1860 & 2 & net \\
\hline \multicolumn{3}{|l|}{ Subfamily Micronycterinae } & Dermanura gnoma (Handley, 1987) & 6 & net \\
\hline Micronycteris microtis Miller, 1898 & 2 & net & Mesophylla macconnelli Thomas, 1901 & 1 & net \\
\hline \multicolumn{3}{|l|}{ Subfamily Lonchorhininae } & $\begin{array}{l}\text { Platyrrhinus angustirostris Velazco, Gardner \& } \\
\text { Patterson, } 2010\end{array}$ & 3 & net \\
\hline Lonchorhina inusitata Handley \& Ochoa, 1997* & 2 & net & Uroderma bilobatum Peters, 1866 & 2 & net \\
\hline \multicolumn{3}{|l|}{ Subfamily Phyllostominae } & Vampyressa thyone Thomas, 1909 & 1 & net \\
\hline Chrotopterus auritus (Peters, 1856) & 1 & net & \multicolumn{3}{|l|}{ Family Mormoopidae } \\
\hline Lophostoma silvicolum D’ Orbigny, 1836 & 6 & net & \multirow{2}{*}{ Pteronotus parnellii (Gray, 1843) } & \multirow{2}{*}{4} & \multirow{2}{*}{$\begin{array}{l}\text { roost (hollow } \\
\text { logs) }\end{array}$} \\
\hline Phylloderma stenops (Peters, 1865) & 2 & net & & & \\
\hline Phyllostomus hastatus (Pallas, 1767) & 2 & net & Pteronotus personatus (Wagner, 1843) & 1 & $\begin{array}{l}\text { roost (hollow } \\
\text { logs) }\end{array}$ \\
\hline Phyllostomus elongatus (Geoffroy, 1810) & 1 & net & \multicolumn{3}{|l|}{ Family Noctilionidae } \\
\hline Trachops cirrhosus (Spix, 1823) & 1 & net & \multirow{2}{*}{ Noctilios albiventris Desmarest, 1818} & \multirow[t]{2}{*}{2} & net \\
\hline Tonatia saurophila Koopman \& Williams, 1951* & 1 & net & & & \\
\hline Subfamily Glossophaginae & & & Muntis niaricans (Genffroy 1824) & 4 & roost (hollow \\
\hline Glossophaga soricina (Pallas, 1766) & 3 & net & Myotis nigricans (Geoftroy, 1824) & 4 & logs) \\
\hline
\end{tabular}

* First record for the Mato Grosso State

Table 2. Localities, habitats, and bat species collected in the Juruena National Park, northern Mato Grosso State.

\begin{tabular}{|c|c|c|c|}
\hline Locality name & Coordinates & Habitat & Bat species \\
\hline $\begin{array}{l}\text { Prainha - Left margin of Juruena } \\
\text { River (camping) }\end{array}$ & 8॰54'32.0”S 58॰33'27.6”W & Ombrophilous dense forest & $\begin{array}{c}\text { M. microtis, L. silvicolum, P. stenops, } T \text {. cirrhosus, } \\
\text { C. perspicillata, A. lituratus, } P \text {. parnellii, and } P . \\
\text { personatus }\end{array}$ \\
\hline Right Margin of Juruena River & $8^{\circ} 54^{\prime} 14.9^{\prime \prime} \mathrm{S} 58^{\circ} 33^{\prime} 30.2^{\prime \prime} \mathrm{W}$ & Ombrophilous dense forest & C. perspicillata and $A$. concolor \\
\hline Serra do Apiacás & 8॰56'17.4"S 58॰33'39.6”W & Ombrophilous dense forest & $\begin{array}{l}\text { P. macrotis, S. leptura, P. hastatus, G. soricina, } \\
\text { C. perspicillata, C. trinitatum, M. macconnelli, P. } \\
\text { angustirostris, and U. bilobatum }\end{array}$ \\
\hline Ilha do Juruena & 8०53'25.0"S 58³3'47.4”W & Ombrophilous dense forest & C. perspicillata \\
\hline São João River trail & 8॰57'02.7"S 58॰32'41.4”W & $\begin{array}{l}\text { Ombrophilous dense forest/open } \\
\text { forest }\end{array}$ & $\begin{array}{l}\text { P. leucoptera, } R \text {. naso, S. bilineata, L. inusitata, } \\
\text { L. silvicolum, A. lituratus, } N \text {. albiventris, and } M \text {. } \\
\text { nigricans }\end{array}$ \\
\hline Murilândia & $9^{\circ} 00^{\prime} 43.3^{\prime \prime} \mathrm{S} 58^{\circ} 36^{\prime} 02.1^{\prime \prime} \mathrm{W}$ & $\begin{array}{l}\text { Ombrophilous dense forest/open } \\
\text { forest }\end{array}$ & None \\
\hline Cedro trail & $8^{\circ} 50^{\prime} 57.5^{\prime \prime} \mathrm{S} 58^{\circ} 28^{\prime} 17.1^{\prime \prime} \mathrm{W}$ & $\begin{array}{l}\text { Ombrophilous dense forest/open } \\
\text { forest }\end{array}$ & $\begin{array}{l}\text { C. auritus, } P \text {. elongatus, } T \text {. saurophila, and } A \text {. } \\
\text { obscurus }\end{array}$ \\
\hline Campinarana & $8^{\circ} 47^{\prime} 51.3^{\prime \prime} \mathrm{S} 58^{\circ} 26^{\prime} 03.5^{\prime \prime} \mathrm{W}$ & $\begin{array}{l}\text { Ombrophilous dense forest/open } \\
\text { savannah }\end{array}$ & C. villosum \\
\hline
\end{tabular}




\begin{tabular}{|c|c|c|c|}
\hline Locality name & Coordinates & Habitat & Bat species \\
\hline Casa de Palha (Camping) & $8^{\circ} 08^{\prime} 41.8^{\prime \prime S} 58^{\circ} 17^{\prime} 51.4^{\prime \prime W}$ & $\begin{array}{l}\text { Ombrophilous dense forest/ } \\
\text { forested savannah }\end{array}$ & $\begin{array}{c}\text { P. kappleri, P. macrotis, L. silvicolum, C. perspicillata, } \\
\text { and A. planirostris }\end{array}$ \\
\hline São Tomé River, right margin & $8^{\circ} 12^{\prime} 06.0^{\prime \prime S} 58^{\circ} 11^{\prime} 18.1^{\prime \prime W}$ & $\begin{array}{l}\text { Ombrophilous dense forest/open } \\
\text { forest/forested savannah }\end{array}$ & C. perspicillata and A. planirostris \\
\hline São Tomé River, left margin & 8॰12'27.2”S 58॰12'11.3”W & $\begin{array}{l}\text { Ombrophilous open forest/ } \\
\text { forested savanna/open savannah }\end{array}$ & C. perspicillata and P. parnellii \\
\hline Juruena River, manioc plantation & $8^{\circ} 06^{\prime} 44.1^{\prime \prime S} 58^{\circ} 17^{\prime} 20.1^{\prime \prime W}$ & $\begin{array}{l}\text { Ombrophilous dense forest/open } \\
\text { forest/forested savannah }\end{array}$ & L. silvicolum, P. hastatus, and $A$. concolor \\
\hline São Simão Archaeological Site & 8॰13'28.3”S 58¹9'02.4”W & $\begin{array}{l}\text { Fluvio-lacustrine vegetation/ } \\
\text { forested savannah }\end{array}$ & None \\
\hline Camping & $7^{\circ} 36^{\prime} 57.2^{\prime \prime} \mathrm{S} 57^{\circ} 56^{\prime} 54.6^{\prime \prime} \mathrm{W}$ & $\begin{array}{l}\text { Ombrophilous dense forest/open } \\
\text { forest }\end{array}$ & $\begin{array}{l}\text { L. silvicolum, C. benkeithi, C. perspicillata, and } A . \\
\text { planirostris }\end{array}$ \\
\hline Taperinha Igapó & 7³9’24.3”S 5756’26.9”W & $\begin{array}{l}\text { Ombrophilous dense forest/open } \\
\text { forest }\end{array}$ & None \\
\hline Morrote & 7³4’05.1”S 5757’56.2”W & $\begin{array}{l}\text { Ombrophilous dense forest/open } \\
\text { forest }\end{array}$ & P. kappleri, A. planirostris, and V. thyone \\
\hline Fruit plantation (camping) & $7^{\circ} 17^{\prime} 19.5^{\prime \prime} \mathrm{S} 58^{\circ} 10^{\prime} 38.9^{\prime \prime} \mathrm{W}$ & $\begin{array}{l}\text { Ombrophilous dense forest/open } \\
\text { forest }\end{array}$ & $\begin{array}{c}\text { C. benkeithi, C. brevicauda, } A \text {. obscurus, } A \text {. } \\
\text { planirostris, and U. bilobatum }\end{array}$ \\
\hline $\begin{array}{l}\text { Surucucu, Vila de Colares } \\
\text { (camping) }\end{array}$ & $6^{\circ} 58^{\prime} 41.9^{\prime \prime} \mathrm{S} 58^{\circ} 21^{\prime} 56.6^{\prime \prime} \mathrm{W}$ & $\begin{array}{l}\text { Ombrophilous open forest/ } \\
\text { forested savanna }\end{array}$ & C. brevicauda and $A$. planirostris \\
\hline Taboca Road (Trilha do Garimpo) & 658'14.9”S 58²3’38.0”W & $\begin{array}{l}\text { Ombrophilous open forest/ } \\
\text { forested savanna }\end{array}$ & $P$. kappleri and $P$. macrotis \\
\hline
\end{tabular}

Grosso, and making it one of the most effective strategies in controlling deforestation and conservation of Amazonian ecosystems (BRASIL 2004). In this sense, estimation of diversity and other basic information on fauna can be useful for conservation purposes, and despite the low sampling effort, which is common in Rapid Ecological Assessments, species richness was relatively high, and the highest for a single study site in Mato Grosso, to date. These results reinforce the need for inventories in areas with gaps in biogeographical and ecological knowledge, such as southern Amazonia. Furthermore, this enhances the role of PNJu for biodiversity conservation in the Southern Amazon.

\section{ACKNOWLEDGMENTS}

This study forms part of the Programa Áreas Protegidas da Amazônia (ARPA) initiative towards the management plans of Amazon protected areas, conducted by Ministério do Meio Ambiente (MMA), Instituto Chico Mendes de Conservação da Biodiversidade (ICMBio), with the involvement of Fundo Global para o Meio Ambiente (GEF), Banco Mundial, Banco de Cooperação do Governo da Alemanha (KfW), Agência de Cooperação Alemã (GTZ), World Wildlife Found (WWF) Brasil, and Fundo Brasileiro para a Biodiversidade (FUNBIO). We thank Paul Velazco for specimens identification, Burton K. Lim and two anonymous referees for criticism on the first versions of the manuscript. This study is partially supported by $\mathrm{CNPq}$ (RG).

\section{REFERENCES}

Bernard, E.; Aguiar, L.M.S.; Machado, R.B. 2011a. Discovering the Brazilian bat fauna: A task for two centuries? Mammal Review, 41: 23-39.

Bernard, E.; Tavares, V.C.; Sampaio, E. 2011b. Compilação atualizada das espécies de morcegos (Chiroptera) para a Amazônia brasileira. Biota Neotropica, 11: 35-46.

Brasil. 2004. Plano de Ação para a Prevenção e Controle do Desmatamento na Amazônia Legal. Casa Civil. Brasília, 156p.

Ferreira, J.; Aragão, L.E.O.; Barlow, J.; Barreto, P.; Berrenger, E.; Bustamante, M.; et al. 2014. Brazil's environmental leadership at risk. Mining and dams threaten protected areas. Science, 346 : 706-707.

Gardner, A.L. 2008. Mammals of South America. Vol. 1, Marsupials, Xenarthrans, Shrews, and Bats. University of Chicago Press, Chicago, $\mathrm{xx}+666 \mathrm{p}$.

ICMBio, 2011. Plano de Manejo do Parque Nacional do Juruena. http://www./portal/images/icmbio.gov.br/portal/images/ stories/imgs-unidades-coservacao/Encarte2.pdf. Accessed on 18/04/2015.

Lim, B.K.; Engstrom, M.D.; Ochoa, G.J. 2005. Mammals. In: Hollowell T. and R.P. Reynolds (Ed.). Checklist of the terrestrial vertebrates of the Guiana Shield Bulletin of the Biological Society of Washington, 13: 77-92.

Louzada, N.S.V.; Monte Lima, A.C.; Pessôa L.M.; Cordeiro, J.L.P.; Oliveira, L.F.B. 2015. New records of phyllostomid bats for the state of Mato Grosso and for the Cerrado of Midwestern Brazil (Mammalia: Chiroptera). Check List, 11(article 1644): 1-10.

MDIC. 2012. Subsidios e proposiçōes para a formulação de uma política industrial sustentável na Amazônia. Sumários Executivos: Produtos 
2-3-4-5. Ministério do Desenvolvimento, Indústria e Comércio Exterior, 153p.

Miranda, J.M.D.; Zago, L.; Runio, M.B.G.; Bernardi, I.P. 2015. Morcegos (Mammalia: Chiroptera) da regiáo do médio Rio Teles Pires, sul da Amazônia, Brasil. Acta Amazonica, 45: 89-100.

Miranda-Ribeiro, A. 1914. Zoologia. Comissão de Linhas Telegráficas e Estratégicas de Matto Grosso ao Amazonas. Hoehne, Rio de Janeiro 17 (Anexo 5): 1-49.

Nogueira, M.R.; Lima, I.P.; Moratelli, R.; Tavares, V.C.; Gregorin, R.; Peracchi, A.L. 2014. Checklist of Brazilian bats, with comments on original records. Check List, 10: 808-821.

Santos, R.R.; Irgang, G.V. 2008. Relatório do Meio Físico para o Plano de Manejo do Parque Nacional do Juruena. Estados de Mato Grosso e Amazonas. Instituto Centro de Vida. WWF-Brasil. Instituto Chico Mendes de Conservação da Biodiversidade, 324p.
Sikes, R.S.; Gannon, W.L.; Animal Care and Use Committee of the American Society of Mammalogists. 2011Guidelines of the American Society of Mammalogists for the use of wild mammals in research. Journal of Mammalogy, 92: 235-253.

Simmons, N.B.; Voss, R.S. 1998. The mammals of Paracou, French Guiana: a Neotropical lowland rainforest fauna part I. Bats. Bulletin of the American Museum of Natural History, 237: 1-219.

Velazco, P.M.; Gardner, A. L.; Patterson, B.D. 2010. Systematics of the Platyrhinus helleri species complex (Chiroptera: Phyllostomidae) with descriptions of two new species. Zoological Journal of the Linnean Society, 159: 785-812.

Recebido em 12/03/2015

Aceito em 04/09/2015 


\section{APPENDIX 1 - LIST OF VOUCHER SPECIMENS THAT ARE FLUID-PRESERVED AND HOUSED AT CMUFLA.}

Artibeus concolor: 1296; Artibeus lituratus: 1286, 1291, 1300, 1303, 1306-1308, 1311, 1312; Artibeus obscurus: 1287, 1288, 1301, 1302; Artibeus planirostris: 1279, 1280, 1286, 1292, 1294; Carollia benkeithi: 1238, 1239; Carollia brevicauda: 1242, 1246, 1257-1259; Carollia perspicillata: 1240, 1241, 1243-1245, 1247-1256; Chiroderma trinitatum: 1284; Chiroderma villosum: 1290, 1299; Chrotopterus auritus: 1270; Dermanura gnoma: 1278, 1281, 1283, 1293, 1295, 1304; Glossophaga soricina: 1321-1323; Lophostoma silvicolum: 1260, 1261, 1263, 1271, 1273, 1275; Lonchorbina inusitata: 1264, 1265; Mesophylla macconnelli: 1297; Micronycteris microtis: 1266; Myotis nigricans: 1324-1326; Noctilio albiventris: 1313, 1314; Peropteryx kappleri: 1218, 1221, 1226; Peropteryx leucoptera: 1320; Peropteryx macrotis: 1220, 1222-1225, 1233, 1234; Phylloderma stenops: 1276, 1277; Phyllostomus elongatus: 1274; Phyllostomus hastatus: 1267, 1272; Platyrrhinus angustirostris: 1298, 13009, 1310; Pteronotus parnelli: 1315, 1316, 1318, 1319; Pteronotus personatus: 1317; Rhinchonycterys naso: 1227-1229; Rhinophylla pumilio: 1236, 1237; Saccopteryx bilineata: 1217, 1230-1232, 1235; Saccopteryx leptura: 1327; Tonatia saurophila: 1269; Trachops cirrhosus: 1262; Uroderma bilobatum: 1282, 1305; Vampyressa thyone: 1285. 